\title{
Taking the "text" out of context effects in repetition priming of word identification
}

\author{
MICHAEL E. J. MASSON \\ University of Victoria, Victoria, British Columbia, Canada \\ and \\ COLIN M. MACLEOD \\ University of Toronto, Scarborough, Ontario, Canada
}

\begin{abstract}
Repetition priming of masked word identification is reduced when initial exposure to target words is in a text rather than in a word list. We demonstrate that there is nothing special about the text context that reduces priming. In Experiment 1, target words read in normal text or in rapid serial visual presentation (RSVP) text-either coherent or scrambled-produced similarly reduced priming, relative to the same words read aloud in a list. In Experiment 2, the delay was decreased between study and test for words presented in text, but they still displayed less priming than did words presented in a study list and tested after an equivalent delay. In Experiment 3, presenting study list words in RSVP to prevent reading each word aloud diminished priming to the same level as that in the text context. We conclude that presenting a target in context prevents it from being encoded and responded to as distinctively as when presented in isolation.
\end{abstract}

Processing episodes have been shown to have persistent and highly specific effects on subsequent task performance, even in the absence of conscious efforts to remember the original episodes. A hallmark illustration of this phenomenon is the enhanced rereading of geometrically transformed text in the studies of Kolers (1975). This specificity of processing principle is perhaps best exemplified in the repetition priming of words, in which prior exposure to a word increases the speed or the accuracy of subsequent processing of that word (e.g., Scarborough, Cortese, \& Scarborough, 1977). As a consequence, repetition priming has been a fertile testing ground for theories of word identification and memory (for a review, see Tenpenny, 1995).

We now know that repetition priming is constrained by a number of factors, such as modality of presentation (e.g., Kirsner, Milech, \& Standen, 1983), that determine the size of the repetition effect and indeed whether it is obtained at all. In addition to modality, the context in which a word appears during its initial presentation also strongly influences repetition priming (see Tenpenny,

This research was supported by Natural Sciences and Engineering Research Council of Canada Research Grants A7910 and A7459 to M.E.J.M. and C.M.M., respectively. The authors thank Judy Caldwell, Carrie Hicks, and Shelley Hodder for their assistance with data collection and Betty Ann Levy and an anonymous reviewer for helpful comments on an earlier version of this article. Correspondence should be addressed to M. E. J. Masson, Department of Psychology, University of Victoria, P. O. Box 3050, Victoria, BC, V8W 3P5 Canada (e-mail: mmasson@uvic.ca), or to C. M. MacLeod, Division of Life Sciences, University of Toronto at Scarborough, Scarborough, ON M1C 1A4, Canada (e-mail:macleod@scar.utoronto.ca).
1995, pp. 348-354). In particular, Levy and Kirsner (1989; see also Oliphant, 1983) found no reliable repetition priming when target words first appeared as part of meaningful text, then appeared in isolation on a masked word identification test. Levy and Kirsner argued from this result that processing operations involved in text comprehension do not transfer across linguistic levels, from words studied in text to words tested in isolation.

Other studies, however, have shown that at least some priming can be produced on a word identification task after reading target words embedded in text (Bourassa, Levy, Dowin, \& Casey, 1998; MacLeod, 1989; Nicolas, 1996, 1998; Wippich \& Mecklenbraeuker, 1995). These studies suggest that the amount of repetition priming produced by words originally read in text context depends on a variety of factors. For example, Nicolas (1996) noted that he used low-frequency words (unlike Levy \& Kirsner, 1989) and suggested that priming from words embedded in text context might be more likely with that type of item. Alternatively, priming appears to be more likely when a target word embedded in text does not fit meaningfully with the text (MacLeod, 1989) or when subjects have relatively low reading skill so that text comprehension becomes secondary to attention-demanding word identification processes (Bourassa et al., 1998).

Even though repetition priming is sometimes found with words that previously appeared in a text context, that priming effect has consistently been shown to be less than the amount of priming found when target words are instead presented in a study phase as an unrelated list of words (Levy \& Kirsner, 1989; Oliphant, 1983) or in a manner that makes the target words stand out from their context (MacLeod, 1989). The purpose of the present 
study was to determine why embedding words in a meaningful text context reduces repetition priming.

We considered a number of factors that distinguish reading a word in text from reading that same word in isolation. First, viewing words in a full text provides a lexical, syntactic, and semantic setting for the words, in the form of a coherent message. Second, when target words are embedded in a text, they are surrounded by substantial visual context, whether or not that context is encoded as a meaningful text. Third, reading a set of texts prior to performing the word identification test creates a longer delay between the initial exposure event and the subsequent test event. Fourth, in a text, the words are in a way "de-individuated," in that they are not presented as discrete events, nor are they usually responded to individually, thereby reducing their distinctiveness. Any one or more of these factors might contribute to the reduced repetition priming seen following presentation in a text context as opposed to a word list context. Our goal was to test these alternative possibilities by varying the nature of the context in which target words were initially presented and by using a single, constant measure of repetition priming.

\section{EXPERIMENT 1}

In Experiment 1, we tested whether embedding target words in coherent text or simply in a visual context that would not be present at test determined the level of priming on a masked word identification test. Target words were presented in one of four context conditions. In the full text condition, the subjects silently read a set of short texts for comprehension, with each text containing two target words. In the RSVP text condition, rapid serial visual presentation (RSVP) was used, in which each word of the text appeared individually at the center of a computer monitor for a fixed duration, then was replaced by the next word of the text. In the RSVP scrambled condition, the words of the texts were randomly reordered, and lists of unrelated words, approximately equal in length to the texts, were formed. These scrambled texts were presented using the RSVP method and were read silently, as in the RSVP text condition. Finally, in the word list condition, 40 target words were presented in random order to be read aloud one by one.

In the test phase, the subjects performed a masked word identification task, in which each target word appeared briefly and was followed by a mask. The task was to identify each word. Some of the target words had appeared in the study phase, and others were new. If viewing the entire text simultaneously - as in normal reading -is the critical factor, then only the full text condition should show reduced priming. If embedding words in a coherent text is responsible for reducing repetition priming, only the full text condition and the RSVP text condition should show reduced priming. If reduced priming is caused by failure to encode and respond to a target as a distinct event, then all three text conditions should yield similarly reduced priming.

\section{Method}

Subjects. Eighty undergraduate students at the University of Victoria participated in the experiment for extra credit in their introductory psychology course. One additional subject was excluded because of language difficulty. Twenty subjects were randomly assigned to each group.

Apparatus. Instructions and materials were presented using a Macintosh II microcomputer with two monitors. The materials were shown to the subject on the primary monitor; the secondary monitor displayed trial information to the experimenter. Words appeared as black letters on a white background. A six-key button box allowed the subject to initiate the trials and to respond to the comprehension test given after reading the texts. For the masked word identification task, the experimenter recorded the subject's accuracy on each trial using the keyboard.

Materials. There were 80 critical words, varying in length from four to six letters. They ranged in frequency from 1 to 547 occurrences per million, with a median frequency of 53 and a mean frequency of 78 (Kučera \& Francis, 1967). In addition, there were 40 texts, each approximately 100 words long and containing two of the critical words. The texts were designed to be factual and interesting and were created using various nonfiction sources. For each text, a single true-false comprehension statement was created to be presented subsequent to the text to ensure that the subjects had read the texts. For counterbalancing purposes, the 40 texts were divided into two blocks of 20 , with the critical words divided correspondingly in constructing the word list.

Design. For counterbalancing purposes, the subjects were shown 40 of the 80 critical words in the study phase. These words were presented in one of four conditions: (1) full text (words embedded in meaningful texts presented in page format), (2) RSVP text (words embedded in meaningful texts presented using RSVP), (3) RSVP scrambled (words embedded in randomized texts presented using RSVP), or (4) word list (words presented in isolation in the form of a list).

In the full text condition, the entire text was presented on the screen, and the subject was allowed to read silently at his or her own pace. In the RSVP text condition, the text was presented one word at a time at the center of the screen. In the RSVP scrambled condition, all of the words from all of the texts were randomized, and then blocks of approximately 100 words (including two critical words) were selected to create the new scrambled texts. All words were to be read silently in these two RSVP conditions. In the word list condition, only the 40 critical words were shown, and the subjects were instructed to read each word aloud to ensure encoding.

Following the study phase, each subject completed two masked word identification test phases. A pretest phase was used to determine for each subject a contrast level to use in the display of words in the test phase. In the test phase proper, the subjects were presented with all 80 of the critical words. Half of these words had appeared in the study phase (old), and half had not (new). The pretest and test phases were identical across the four study conditions.

Procedure. After an initial instruction screen, the word "READY" was displayed at the center of the subject's monitor until a keypress, then the screen was blanked for $495 \mathrm{msec}$, signaling the start of the study phase. In the text conditions, the 20 texts were then presented one at a time in random order. In the full text condition, the entire text was displayed on the subject's monitor and remained there until a keypress. The comprehension statement for that text was then presented, and the subject responded "yes" or "no" by pressing either 
the right or the left key, respectively, on the response box. In the RSVP conditions (text or scrambled), each text was displayed one word at a time, with each word appearing at the center of the subject's monitor for $105 \mathrm{msec}$, followed by a 255 -msec blank screen. After the last word in a text, there was a $495-\mathrm{msec}$ blank screen. For the RSVP text condition, the comprehension statement for that text was then presented. For the RSVP scrambled condition, a one-item recognition test followed the text. For half of the scrambled texts, this was a noncritical word taken from the text; for the other half, this was a filler word not taken from any text. The subjects responded as in the full text condition.

In the word list condition, four filler words were presented in random order, followed by the 40 critical words. These words were presented one at a time in the center of the subject's monitor. Each word remained on the screen until the subject read it aloud and the experimenter pressed the space bar, after which the word was erased. A 495 -msec blank screen preceded the next word. There was no comprehension or recognition test in this condition.

For all four conditions, the pretest phase began with an instruction screen and four practice trials. The practice words were presented at maximum contrast at the following decreasing durations: $75,60,45$, and $30 \mathrm{msec}$, with $30 \mathrm{msec}$ representing the presentation duration of the rest of the pretest items and of the critical items. Following the last practice word, the pretest trials were presented. Each trial began with the word "READY." After the subject pressed a key, there was a 255 -msec blank screen before two hyphens appeared, separated $n$ by blank spaces, where $n$ was the number of letters in the upcoming word plus two extra spaces, one at each end of the word. This warning was displayed for $255 \mathrm{msec}$ before the word appeared in lowercase letters between the hyphens. After a 30-msec exposure, the word was immediately replaced by a mask consisting of a row of ampersands equal in length to the warning stimulus. The mask remained on the screen for $150 \mathrm{msec}$ and was followed by a series of eight question marks, which stayed on the screen until the subject made an oral response. The experimenter recorded the correctness of the subject's response by pressing an appropriate key on the keyboard.

Over the course of up to 40 pretest trials, the contrast level used to display the word was automatically adjusted using the PEST procedure (Pentland, 1980). The goal of this adjustment was to reach a contrast value at which the subject maintained $60 \%$ identification accuracy. When this occurred, the pretest ceased; if this did not occur, all 40 pretest trials were completed.

The test phase began immediately after the pretest phase was completed. Here, each of the 80 critical words was presented individually for masked word identification, with all of the trials conducted at the contrast value determined in the pretest phase. Otherwise, all other aspects of the test procedure were identical to the pretest procedure. The order of testing the critical words was randomized, and no feedback was provided as to identification accuracy.

\section{Results and Discussion}

The mean proportions of correctly answered comprehension statements in the study phase were $.89(S E=$ $.03)$ and $.85(S E=.02)$ for the full text and RSVP text conditions, respectively, with all subjects performing above .50. The mean proportion correct on the recognition test used during the study phase in the RSVP scrambled condition was $.68(S E=.03)$, with all but 1 subject performing above chance. These data suggest that the subjects were attending to the materials presented during the study phase, although the subjects in the RSVP scrambled condition found the recognition test rather challenging.

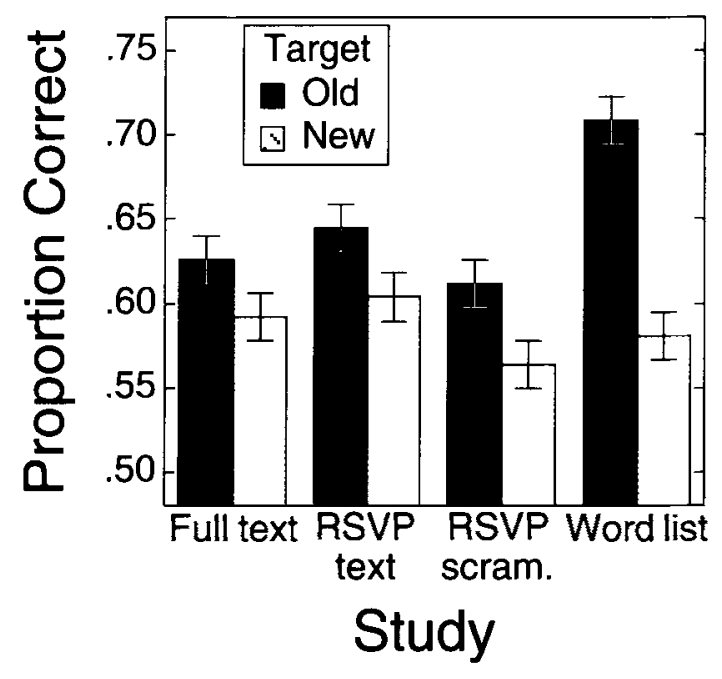

Figure 1. Mean proportion correct in the masked word identification task of Experiment 1. Error bars are $95 \%$ within-subjects confidence intervals and are appropriate for comparing performance on old and new targets.

The mean proportion of correct responses for each study condition on the masked word identification task is shown in Figure 1. The pattern of priming effects is clear: The full text, RSVP text, and RSVP scrambled conditions all produced about the same amount of priming, which was less than the priming seen in the word list condition. A $4 \times 2$ mixed analysis of variance (ANOVA), with study condition between subjects and target status (old vs. new) within subjects, was computed to verify this interpretation of the data. A Type I error rate of .05 was used in this and all statistical tests reported in this article.

The ANOVA yielded a significant main effect of target status, with more correct responses to old words than to new words $\left[F(1,76)=37.89, M S_{\mathrm{e}}=0.004\right]$, although the main effect of study condition was not significant $(F<1)$. The interaction between study condition and target status was significant $\left[F(3,76)=4.74, M S_{\mathrm{e}}=0.004\right]$. To interpret the interaction, two comparisons were conducted on the priming scores (old - new). The first comparison showed that the mean priming score averaged across the full text, the RSVP text, and the RSVP scrambled conditions (.04) was significantly lower than the priming score in the word list condition $(.13)[F(1,76)=$ $\left.13.98, M S_{\mathrm{e}}=0.008\right]$. The second comparison showed that the priming scores for the first three conditions were not significantly different from each other $(F<1)$. Mean priming across these three conditions was, however, significantly greater than zero $[t(59)=3.73, S E M=0.011]$.

The results clearly indicate that neither the visual context of normally presented text nor the linguistic processing associated with connected text are necessarily involved in the reduction of repetition priming for target words selected from a previously viewed text. Repetition 
priming suffered equally, regardless of the nature of the larger context in which target words were embedded. A much larger priming effect was observed when the targets were entirely removed from background material and presented as a short list of unrelated words. These results suggest a modification to the proposal that reduced repetition priming associated with text context is a result of failed transfer between processing tasks aimed at different linguistic levels represented by text versus individual words (Levy \& Kirsner, 1989). Namely, the "text" level should be broadened to include contexts, such as those afforded by scrambled texts, that are less globally coherent. Whittlesea's (1990) demonstration of the contextual influence of scrambled texts on word reading is consistent with this suggestion.

\section{EXPERIMENT 2}

Although Experiment 1 provided strong evidence against a special role for coherent text or visual context in reducing repetition priming relative to the word list condition, three other factors were not addressed by that experiment. First, the two text conditions and the RSVP scrambled condition all involved presentation of 20 sets of approximately 100 words each in the study phase, followed by the test phase. Thus, the studied target items were embedded in a relatively large nominal study list of about 2,000 words. This list was much larger than the 40word list used in the word list condition. Second, because the test phase was not given until all study lists had been presented, the subjects in the three text conditions experienced a relatively long retention interval, on average, between the time a target word appeared in its study context and the time it appeared on the masked word identification test. Third, the subjects in the word list condition read aloud each target word, whereas the subjects in the other three conditions read silently. Reading a word aloud - in whatever context - may be sufficient to produce the large priming effect seen in the word list condition, perhaps because of additional attention or processing time devoted to a word when it is read aloud relative to when it is read silently.

To differentiate these three factors as possible causes of differential priming, in Experiment 2, we modified the method of testing words presented in text contexts. Two study conditions were used: full text and word list. The procedure for the word list condition was the same as in Experiment 1. For the full text condition, however, texts were reduced to about 50 words in length, and there was a masked word identification test after each text was read. This test included two practice words, two critical words taken from the text that was just read, and two new words. Thus, both the size of the nominal study list and the length of the study-test retention interval in the full text condition were comparable to those in the word list condition. Moreover, the subjects in the full text condition read each text aloud, just as did the subjects in the word list condition.
If greater study list length, longer study-test retention interval, or silent reading is responsible for preventing priming of targets embedded in a text context from reaching the level of priming seen in the word list condition, then Experiment 2 should find equal priming in the full text and word list conditions. On the other hand, if encoding targets as distinct events, isolated from background events, is critical, then greater priming should again be found in the word list condition.

\section{Method}

Subjects. Forty-eight undergraduate students at the University of Toronto at Scarborough participated either for bonus points in their introductory psychology course or for payment (\$5). The data of 2 additional subjects were discarded due to language difficulties; those of 3 further subjects were discarded because of performance at floor, defined as zero correct for either the set of old words or the set of new words. Four other subjects participated only in the practice phase of the experiment but elected not to continue beyond that point. Twenty-four subjects were randomly assigned to each of the two study conditions described below.

Apparatus. The experiment was controlled by an IBM-AT compatible microcomputer with a color monitor. Displays were in black on a white background.

Materials. The 80 critical words ranged in frequency from 0 to 897 occurrences per million, with a median frequency of 62 and a mean frequency of 116 (Kučera \& Francis, 1967). An additional 6 words were selected for practice in the masked word identification test phase. A further 24 high-frequency words were used in the pretest phase.

Twenty critical texts and one practice text were constructed, each about 50 words in length. A true-false comprehension statement was constructed for each critical text. There were two versions of each critical text, as illustrated in the example in Table 1. Version 1 contained two critical words (e.g., data and speed) and two synonyms corresponding to the critical words used in Version 2 (aircraft and thaw). In Version 2, the two critical words ( plane and melt) replaced the synonyms from Version 1, and two synonyms (information and velocity) replaced the critical words from Version 1. This arrangement permitted counterbalancing of old and new words at study and test. Both versions also contained a word used for practice (radar), and there was another practice word consistent with the text but not actually included in it ( frost). Critical words in the word lists were identical to those used in the texts.

\section{Table 1}

\section{Sample Text and Target Words Used in Experiment 2}

\section{Version 1}

Navy pilots often cover 15,000 square miles of ocean, plotting the location of every iceberg they sight or detect with radar. When each aircraft lands, the data goes to Governor's Island. There, every iceberg is given a number, and its thaw rate, direction, and speed of drift are projected.

Practice items: radar (in text) frost (not in text)

Critical items: data speed (in text) plane melt (not in text; replaced by aircraft thaw)

Version 2

Navy pilots often cover 15,000 square miles of ocean, plotting the location of every iceberg they sight or detect with radar. When each plane lands, the information goes to Governor's Island. There, every iceberg is given a number, and its melt rate, direction, and velocity of drift are projected.

Practice items: radar (in text) frost (not in text)

Critical items: plane melt (in text) data speed (not in text: replaced by information velocity) 
Design. There were two study conditions in this experiment: (1) full text (words embedded in meaningful texts presented in page format) and (2) word list (words presented in isolation in the form of a list).

Procedure. All subjects first participated in a practice session to familiarize them with the masked word identification task. Each trial began with a warning ("READY?"), and, when they indicated they were ready, the experimenter initiated the brief presentation of the target word embedded between two hyphens followed by a mask consisting of a row of ampersands. Three blocks of eight trials each were presented. Target duration was adjusted for each subject contingent on performance across these blocks, ranging from 28 to $56 \mathrm{msec}$.

Prior to the study-test phase, the subjects in the full text condition were instructed that they would be shown a series of texts, one at a time, which they were to read aloud at a normal pace so that they could understand the texts. They were also told that they would be tested on the content of all of the texts at the end of the experiment and that, after reading each text, a masked word identification test would be conducted as during the practice phase. The subjects were given feedback on their accuracy for the first study-test cycle, which involved the practice text. The 20 critical study-test cycles immediately followed the practice cycle. Half of the subjects were tested with Version 1 texts and half were tested with Version 2 texts.

Each study-test cycle began with the presentation of the text at the center of the screen. The subject read the text aloud, then completed six masked word identification test trials, using the same procedure as in the practice phase. The six test words consisted of the following (illustrating with Version 1 of the Table 1 materials): one filler that did not occur in the text (frost), one filler that did occur in the text (radar), two critical old words that appeared in the text (data and speed), and two critical new words that did not appear in the text (plane and melt, the critical words from Version 2). Thus, all of the words were relevant to the text. The two filler words were always presented first, with their sequence randomly determined. The four critical old and new words were then presented in random order. After all the critical study-test cycles had been completed, the 20 true-false comprehension statements were presented.

In the study phase of the word list condition, the same critical test words were shown to the subjects as in the full text condition (half of the subjects were presented two critical words from each of the Version 1 texts, and the other half were presented two critical words from the Version 2 texts). Words were presented one at a time at the center of the screen, and the subject read each one aloud. Three filler words were presented prior to the 40 critical words. All words were presented in lowercase. The masked word identification trials for the word list subjects were conducted exactly as in the full text condition. Each subject was presented a sequence of test trials that was identical to a sequence used for a subject in the full text condition. Following the identification test, the 20 true-false statements were presented to the subject as a baseline measure of the ability to guess the answers without having read the texts.

\section{Results and Discussion}

The mean proportion of correctly answered comprehension statements in the full text condition was .80 ( $S E=$ $.03)$; all subjects were above chance, defined as the mean score of subjects in the word list condition $(M=.57$, $S E=.02$ ). This result indicates that the subjects were reading the texts for comprehension, as instructed. The subjects in the word list condition had not read the text and so had to rely on preexperimental knowledge to respond to the statements.

The mean proportions of correct responses on the masked word identification test are shown in Figure 2.

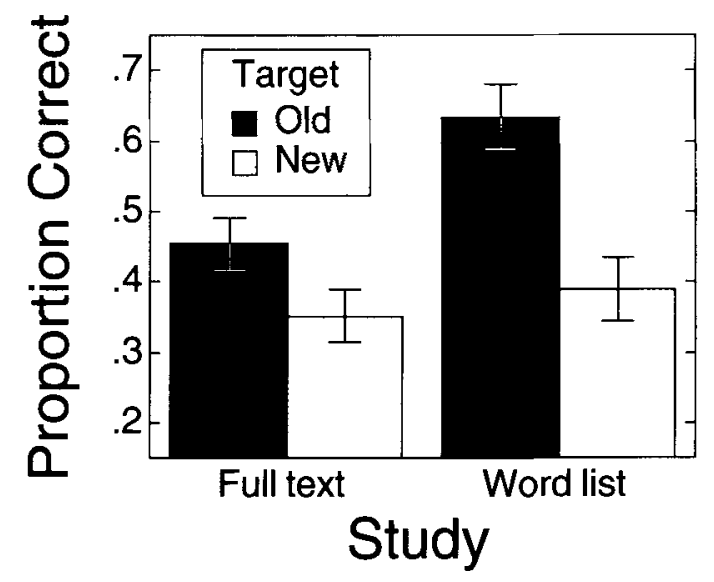

Figure 2. Mean proportion correct in the masked word identification task of Experiment 2 . Error bars are $95 \%$ within-subjects confidence intervals and are appropriate for comparing performance on old and new targets.

Again, the priming effect was substantially greater in the word list condition than in the full text condition. A mixed ANOVA, with study condition (between subjects) and target status (old vs. new; within subjects) as factors revealed no effect of study condition $(p>.10)$ but did reveal a significant target status effect $\left[F(1,46)=73.98, M S_{\mathrm{e}}=\right.$ $0.010]$. There was also an interaction between study condition and target status $\left[F(1,46)=12.63, M S_{\mathrm{e}}=0.010\right]$. The interaction supports the conclusion that repetition priming was significantly greater in the word list condition than in the full text condition, although the priming effect in the full text condition $(M=.10)$ was significantly greater than zero $[t(23)=4.08, S E M=0.025]$. The continued priming advantage for the word list condition in the masked word identification task indicates that none of the three factors addressed by the use of study-test cycles and reading aloud in the full text condition are primarily responsible for the reduced priming seen with text context.

\section{EXPERIMENT 3}

The results of Experiment 2 suggest that embedding words in a text, even when they are read aloud, entails encoding these words as part of the surrounding context. Because that context is not reinstated when targets are presented again in a subsequent masked word identification task, relatively small amounts of repetition priming are seen. As we showed in Experiment 1, however, that context does not have to constitute a coherent text for the encoding to fail to support substantial amounts of priming. In the word list condition, by presenting words as discrete events and requiring the subjects to respond to each, a distinctive encoding that can optimally support later priming seems to have been engaged. We tested this proposal in Experiment 3 by comparing an RSVP text condition, like that used in Experiment 1, with a modified 
word list condition. In the word list condition, words were presented in RSVP format using the same display parameters as in the RSVP text condition. No overt response was made to words presented in either of these conditions.

We expected that presenting the target words as a continuous stream in the word list condition, without providing the subjects an opportunity to make an overt response to each one, would encourage contextually bound encoding of those items. This type of encoding contrasts with the distinctive, individuated encoding assumed to be associated with the word list condition used in Experiments 1 and 2 and in many previous studies of repetition priming that have used the read encoding task (e.g., Jacoby, 1983; Jacoby \& Dallas, 1981; MacLeod \& Masson, 1997; Masson \& MacLeod, 1992). As a result of inducing a contextually bound encoding, we expected repetition priming in the RSVP text condition and in the word list condition of Experiment 3 to be similar. Moreover, we expected that similarity to arise from reduced priming in the word list condition in Experiment 3 relative to the word list condition used in Experiments 1 and 2.

\footnotetext{
Method

Subjects. Forty students from the same pool as Experiment 1 participated. Three additional subjects were not included in the analyses: One found the task too difficult and declined to continue, and the other 2 were at floor (defined as $10 \%$ or less in both conditions) in the masked word identification task. Twenty subjects were randomly assigned to each of the two study conditions.

Apparatus and materials. The equipment and critical stimuli were identical to those used in Experiment 1 .

Design. There were two study conditions: (1) RSVP text (words embedded in meaningful texts presented in RSVP format) and (2) RSVP word list (individual critical words presented in an RSVP list).

Procedure. The RSVP text condition was identical to that in Experiment 1 , with each word appearing on the screen for $105 \mathrm{msec}$, followed by a 255 -msec blank. The RSVP word list condition was a modified version of the word list condition in Experiment 1 , the primary change being the change to RSVP. Two filler items were added to the beginning and to the end of the list. Display timing was the same as in the RSVP text condition. To encourage the subjects to attend to the word list, they were told that their memory for the list would be tested. A two-item recognition memory test was given after presentation of the list, using a randomly selected filler word plus an extra unstudied filler word. The subject's task was to indicate whether each had appeared in the RSVP word list. The pretest and test phases were identical to those used in Experiment 1 .
}

\section{Results and Discussion}

The mean proportion correct on the comprehension statements for the RSVP text condition was $.81(S E=.03)$, and only 1 subject scored below .65. This level of comprehension performance is comparable to that seen in the earlier experiments. In the RSVP word list condition, mean proportion correct on the two-item recognition test was $.75(S E=.07)$, indicating that the subjects generally were attentive to the list.

Figure 3 presents the mean proportions of correct responses on the masked word identification test. It is clear

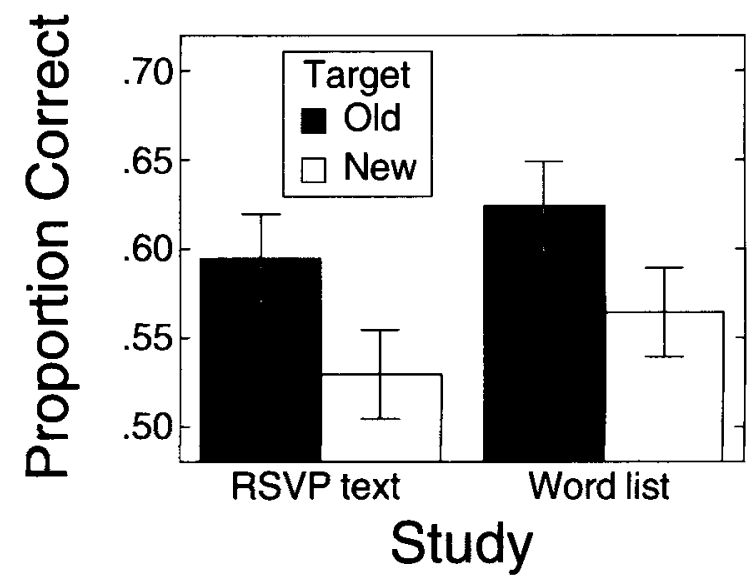

Figure 3. Mean proportion correct in the masked word identification task of Experiment 3. Error bars are $95 \%$ within-subjects confidence intervals and are appropriate for comparing performance on old and new targets.

from this figure that the priming effect was equal in the RSVP text and RSVP word list conditions $(M s=.06)$. An ANOVA, with study condition and target status (old vs. new) as factors, revealed only a significant effect of target status $\left[F(1,38)=11.03, M S_{\mathrm{e}}=0.007\right]$, demonstrating reliable priming. Neither the main effect of study condition nor the interaction approached significance $(F \mathrm{~s}<1)$.

The finding of equal priming for the text and word list conditions stands in sharp contrast to the results of Experiments 1 and 2 . It is consistent, however, with the prediction that removing the response component from the procedure used for the word list condition would bring the magnitude of repetition priming in that condition into line with that seen in a text condition. Indeed, the priming effect in the RSVP word list condition of Experiment $3(M=.06)$ was significantly lower than that found in the word list conditions of Experiments 1 and 2 combined $(M=.19)\left[F(1,62)=12.96, M S_{\mathrm{e}}=0.009\right]$, and was equivalent to the mean priming effect found in the text conditions of Experiments 1 and $2(M=.06)(F<1)$.

\section{GENERAL DISCUSSION}

The original proposal for why repetition priming is weak or absent for words that initially appeared embedded in text (Levy \& Kirsner, 1989; MacLeod, 1989; Oliphant, 1983) was that a text provides an integrating conceptual framework into which words are encoded. This idea has been sustained in more recent work (Nicolas, $1996,1998)$. The argument is that the resulting contextually bound representation of words is not appropriate to support subsequent identification of those words when presented in isolation as targets. On this account, the reduced priming found when words initially appeared in text rather than in an unrelated word list can be explained by invoking the transfer-appropriate processing frame- 
work (e.g., Roediger, Weldon, \& Challis, 1989): Conceptually driven processing involved in reading text does not transfer well to data-driven tests, such as masked word identification.

Our experiments demonstrate that, although contextually bound encoding does appear to be a crucial component in explaining the influence of text context, the involvement of conceptually driven processing invoked by the coherence of that context does not seem to be important. In Experiment 1, priming was reduced just as much when target words were embedded in scrambled texts as in coherent texts, yet scrambled texts do not afford a globally coherent interpretation. That experiment also ruled out a change in general visual context from study (page layout of text) to test (a word in isolation) as a cause of reduced priming, relative to a word list study condition as the benchmark. In Experiment 2, we tested critical words immediately after the subjects read each text aloud, with the goal of bringing the retention interval in the text condition into line with that in the word list condition. Nevertheless, reading words in a text context continued to produce a reduced level of priming on the masked word identification task.

In Experiment 3, we showed that eliminating the requirement to make an overt response to words in the word list condition reduced the degree of repetition priming to the level seen in the text condition. Rather than having the subjects read each word aloud, we had them view the words in a continuous stream without making overt responses to them. Because this procedure ensured that words presented in text or in an unrelated list were seen for equal amounts of time, and because we observed similar amounts of priming for these two conditions, one might conclude that the reduced priming in the text conditions of Experiments 1 and 2 (and in earlier studies) was a function of differences in processing time.

Within limits, however, processing time does not seem to be crucial in determining the size of repetition priming effects. In Experiment 2, the word list condition produced larger priming than the text condition, despite the fact that words were read aloud (and thereby processed for similar amounts of time) in both conditions. Moreover, Masson and Hicks (1999) showed that within the range of durations used in the present experiments, processing time during study does not reliably affect repetition priming on the masked word identification task. In a study phase, they used a search task in which subjects viewed word lists presented in RSVP and attempted to detect the presence of a target word (e.g., blue) defined by a category cue (e.g., COLOR). Words that had been viewed in these RSVP lists but were not targets produced only a small repetition priming effect $(M=.06)$ in a subsequent masked word identification test. That priming effect did not vary systematically as a function of the duration for which words were presented in the RSVP lists, ranging from 200 to $1,000 \mathrm{msec}$ and was virtually the same magnitude as the priming effect seen in the text conditions of the present experiments.
In contrast to repetition priming of nontargets taken from the RSVP lists, Masson and Hicks (1999) found that when detected targets later appeared in a masked word identification task, substantial repetition priming was found $(M=.20)$. They proposed that making an overt response to the target presented as part of the RSVP list allowed it to be encoded as an event distinct from other items in the list and that this distinctive encoding was the foundation for the increased repetition priming.

We suggest that a similar principle is responsible for the reduced priming seen when words initially occur in text context versus in an unrelated list. Namely, integrative of an item together with other items will produce relatively little priming on a subsequent task in which items are tested in isolation. Larger priming will be seen for items originally encoded in a manner that makes them distinct from other items in the encoding context. This contrast between distinctive encoding of an item and encoding in which an item is integrated with its context is similar to that made by Jacoby (1983) and by Masson and MacLeod (1992) and to the distinction between itemspecific and relational encoding (e.g., Hunt \& Einstein, 1981; Hunt \& McDaniel, 1993).

Producing an overt response, however, does not in itself appear to be adequate for generating a large priming effect in masked word identification. In Experiment 2, the subjects read texts aloud, making the overt response of pronunciation to each word in a text, yet a smaller priming effect was obtained in that condition than in the word list condition. Thus, we propose that the overt response made during encoding must be one that allows a word to be distinctively encoded and individuated against the background of other items that are presented. Reading aloud a list of unrelated words, with each displayed in isolation from the rest, appears to meet this criterion: Responding to each word apparently allows each to be encoded as an event distinct from other items in the list. Similarly, reading text in a manner that emphasizes encoding of individual words over text comprehension, either because of instruction (Carlson, Alejano, \& Carr, 1991; Carr, Brown, \& Charalambous, 1989) or because of low reading skill relative to the demands of the text (Bourassa et al., 1998; Faulkner \& Levy, 1994, 1999), can also produce distinctive encoding of a form that supports later fluent identification of words.

In contrast, reading words aloud in a coherent text, although invoking an overt response, is not necessarily adequate to prevent the constituent words from being contextually bound with other items in the text, if the reader is capable of and is oriented toward text comprehension. This idea also neatly accommodates the observation of greater priming for words that do not fit the sentence context in which they are presented than for words that do fit their context (MacLeod, 1989, Experiment 3).

This proposal fits well with our prior work as well as that of others. First, it encompasses the Masson and Hicks (1999) conclusion that making an overt response to an item is important in producing subsequent repetition 
priming. Second, it captures MacLeod's (1989) idea that encoding an item as an event distinct from background events, such as other items, strongly influences later repetition priming. More broadly, it is consistent with the notion of contextual binding promoted by earlier accounts of why repetition priming of words appearing in texts is weak or absent (e.g., Levy \& Kirsner, 1989; MacLeod, 1989). We have shown, however, that there is nothing special about text: Contextual binding sufficient to substantially reduce later repetition priming can be generated even by unrelated word strings (see also Whittlesea \& Brooks, 1988). An important next step in understanding the principles that govern the influence of prior experience on skilled performance is to learn more about the conditions that control contextual binding at encoding.

\section{REFERENCES}

Bourassa, D. C.. Levy, B. A., Dowin, S., \& Casey, A. (1998). Transfer effects across contextual and linguistic boundaries: Evidence from poor readers. Journal of Experimental Child Psychologv, 71, 45-61.

Carlson, L. A., Alejano. A. R., \& Carr, T. H. (1991). The level-offocal-attention hypothesis in oral reading: Influence of strategies on the context specificity of lexical repetition effects. Journal of Experimental Psvchologv: Learning. Memorv. \& Cognition, 17, 924-931.

Carr, T. H., Brown, J. S., \& Charalambous, A. (1989). Repetition and reading: Perceptual encoding mechanisms are very abstract but not very interactive. Journal of Experimental Psychology: Learning. Memorv, \& Cognition, 15, 763-778.

FAULKNER, H. J., \& LEVY, B. A. (1994). How text difficulty and reader skill interact to produce differential reliance on word and content overlap in reading transfer. Journal of Experimental Child Psvchologv, 58, 1-24.

FAULKNER, H. J., \& LeVY, B. A. (1999). Fluent and nonfluent forms of transfer in reading: Words and their message. Psvchonomic Bulletin \& Review, 6, 111-116.

HUNT, R. R., \& EINSTEIN, G. O. (1981). Relational and item-specific information in memory. Journal of Verbal Learning \& Verbal Behavior, 20, 497-514.

HUNT, R. R., \& MCDANIEL. M. A. ( 1993). The enigma of organization and distinctiveness. Journal of Memory \& Language, 32, 421-445.

JACOBY, L. L. (1983). Perceptual enhancement: Persistent effects of an experience. Journal of Experimental Psychology: Learning, Memorv, \& Cognition, 9, 21-38.

JACOBY, L. L., \& Dallas, M. (1981). On the relationship between autobiographical memory and perceptual learning. Journal of Experimental Psychology: General, 110, 306-340.

KirSner, K.. Milecr, D., \& Standen, P. (1983). Common and modalityspecific processes in the mental lexicon. Memory \& Cognition, 11 , $621-630$.
KoLERS, P. A. (1975). Specificity of operations in sentence recognition. Cognitive Psychology, 7, 289-306.

KuČera, J., \& FranCIS, W. N. (1967). Computational analysis of present-day American English. Providence, RI: Brown University Press.

LeVy, B. A., \& Kirsner, K. (1989). Reprocessing text: Indirect measures of word and message level processes. Journal of Experimental Psychology: Learning, Memory, \& Cognition, 15, 407-417.

MACLEOD, C. M. (1989). Word context during initial exposure influences degree of priming in word fragment completion. Journal of Experimental Psychology: Learning, Memory, \& Cognition, 15, 398-406.

MacLeod, C. M., \& Masson, M. E. J. (1997). Priming patterns are different in masked word identification and word fragment completion. Journal of Memory \& Language, 36, 461-483.

Masson, M. E. J., \& HICKS, C. L. (1999). The influence of selection for response on repetition priming of word identification. Canadian Journal of Experimental Psychology, 53, 381-393.

Masson, M. E. J., \& MACLeOD, C. M. (1992). Reenacting the route to interpretation: Enhanced perceptual identification without prior perception. Journal of Experimental Psychology: General, 121, 145-176.

Nicolas, S. (1996). Priming of perceptual identification after word studying in coherent texts: A short report. Current Psychology of Cognition, 15, 309-321.

Nicolas, S. (1998). Perceptual and conceptual priming of individual words in coherent texts. Memory, 6, 643-663.

Oliphant, G. W. (1983). Repetition and recency effects in word recognition. Australian Journal of Psychology, 35, 393-403.

Pentland, A. (1980). Maximum likelihood estimation: The best PEST. Perception \& Psychophysics, 28, 377-379.

Roediger, H. L., III, Weldon, M. S., \& Challis, B. H. (1989). Explaining dissociations between implicit and explicit measures of retention: A process account. In H. L. Roediger III \& F. I. M. Craik (Eds.), Varieties of memory and consciousness: Essays in honour of Endel Tulving (pp. 3-14). Hillsdale, NJ: Erlbaum.

Scarborough, D. L., Cortese, C.. \& Scarborough, H. S. (1977). Frequency and repetition effects in lexical memory. Journal of Experimental Psvchologv: Human Perception \& Performance, 3, 1-17.

TENPENNy, P. L. (1995). Abstractionist versus episodic theories of repetition priming and word identification. Psychonomic Bulletin \& Review, 2, 339-363.

Whittlesea, B. W. A. (1990). Perceptual encoding mechanisms are tricky but may be very interactive: Comment on Carr, Brown, and Charalambous (1989). Journal of Experimental Psychology: Learning, Memory, \& Cognition, 16, 727-730.

Whittlesea, B. W. A., \& Brooks, L. R. (1988). Critical influence of particular experiences in the perception of letters, words, and phrases. Memory \& Cognition, 16, 387-399.

WIPPICH, W., \& MECKLENBRAEUKER, S. (1995). Implicit memory for textual materials. Psychological Research, 57, 131-141.

(Manuscript received September 3, 1999; revision accepted for publication January 12,2000 .) 